\title{
Toxicity of exposure to binary mixtures of four anti-neoplastic drugs in Daphnia magna and Ceriodaphnia dubia
}

\author{
Alfredo Parrella ${ }^{a}$, Michael Kundi ${ }^{b}$, Margherita Lavorgna a ${ }^{\text {a }}$ Emma Criscuolo ${ }^{a}$, \\ Chiara Russo ${ }^{a}$, Marina Isidori ${ }^{\mathrm{a}, *}$ \\ a Dipartimento di Scienze e Tecnologie Ambientali, Biologiche e Farmaceutiche, Seconda Università di Napoli, Via Vivaldi 43, I-81100 Caserta, Italy \\ ${ }^{\mathrm{b}}$ Institute of Environmental Health, Center for Public Health, Medical University of Vienna, Kinderspitalgasse 15, 1090 Vienna, Austria
}

\section{A R T I C L E I N F O}

\section{Article history:}

Received 2 July 2014

Received in revised form

17 September 2014

Accepted 27 September 2014

Available online 7 October 2014

\section{Keywords:}

Anti-neoplastic drugs

Chronic toxicity

Crustaceans

Mixtures

Effluents

\begin{abstract}
A B S T R A C T
Anticancer drugs, interfering with DNA in every living organism, may pose a threat to aquatic environment, even more when they occur as complex mixtures. We investigated the combined long term toxic potential of four anti-neoplastic drugs (5-fluorouracil [5-FU], cisplatin [CDDP], etoposide [ET] and imatinib mesylate [IM]) testing their binary mixtures on two primary consumers of the freshwater aquatic chain with close phylogenetic relationship: Daphnia magna and Ceriodaphnia dubia. The combined toxicities were assessed using two distinct effect sizes that should be observed if Bliss independence holds. Direct statistical comparison by analysis of variance of single and combined toxicities under the assumption of Bliss independence allowed to accept or reject the independency hypothesis. Independency was confirmed for all mixtures both in D. magna and in C. dubia, except for IM+ ET and IM + CDDP in D. magna and for ET + CDDP and ET +5-FU in C. dubia which at the highest concentrations showed an antagonistic interaction. A synergic tendency was found testing IM + CDDP on C. dubia at the lowest concentration selected. Thus, the chronic ecotoxicological data evaluated in this study show not only a potential environmental risk of anticancer drugs, especially considering their potential synergistic effects, but also the necessity to integrate statistical models with experimental data to establish the real environmental impact of such compounds.
\end{abstract}

(c) 2014 Elsevier B.V. All rights reserved.

\section{Introduction}

Pharmaceutical residues found in the aquatic environment, usually occur as mixtures, not as single compounds. The knowledge about toxicological effects of pharmaceutical mixtures is rather limited even if it has been found that they might reveal different effects from those of single compounds (Fent et al., 2006; Galus et al., 2013). The concentrations of pharmaceuticals in the aquatic systems depend on their prevalence of use, human metabolism, possible biotic and/or abiotic transformations, and on the effectiveness of wastewater treatment. Thus, from an environmental point of view, each single drug may be regarded as a chemical mixture (parent compound plus metabolites plus transformation products) (Kümmerer, 2009). In this context, also anti-neoplastic drugs play an important role. Although their concentrations in environmental media are often lower than those of other pharmaceuticals, their potential for interfering with the structure and functions of DNA

\footnotetext{
* Corresponding author. Tel.: +390823 274565.

E-mail address: marina.isidori@unina2.it (M. Isidori).
}

in exposed organisms is likely higher. In addition, the residues of anti-neoplastic drugs rather occur as mixtures because treatment protocols against various cancers are moving towards the administration of a combination of different anticancer drugs to increase treatment efficiency (Shi et al., 2012). Although the concentrations of these drugs in aquatic systems are very low, chronic exposures of organisms may be of environmental concern, especially when they occur as mixtures (Brezovšek et al., 2014). Compounds with the same mode and biological site of action will act in mixtures according to the model of Concentration Addition (CA, Loewe additivity) while substances with independent mechanism and/or different biological sites could act in mixture according to the model of Independent Action (IA, Bliss independence) (Katsnelson et al., 2011). As most of anticancer drugs interfere with DNA, acting on the same biological target but often with different modes of action, it is difficult to predict their interaction in mixtures. For this reason, the aim of the present study was to investigate chronic effects of four anti-neoplastic drugs in binary mixtures on nontarget organisms. The compounds studied were: 5-fluorouracil (5-FU), cisplatin (CDDP), etoposide (ET) and imatinib (IM). These drugs were selected because of their different properties and 
modes of action, belonging to the groups of antimetabolites (5-FU), platinum-derived drugs (CDDP), topoisomerase inhibitors (ET) and kinase inhibitors (IM). The endpoint considered for the toxicity evaluation was the inhibition of the reproduction of two cladoceran crustaceans, Daphnia magna and Ceriodaphnia dubia, already tested with the drugs of interest with EC50 values previously reported (Parrella et al., 2014). This study was designed to establish the effects of these anti-neoplastic drugs in mixtures as compared to those found for single substance exposure. For this purpose, Bliss independence and Loewe additivity approaches were applied according to the Saariselkä agreement (Greco et al., 1992).

\section{Materials and methods}

\subsection{Test compounds}

5-FU (CAS: 51-21-8), CDDP (CAS: 15663-27-1), ET (CAS: 3341942-0) were supplied by Sigma-Aldrich (Milano, Italy). IM (CAS: 220127-57-1) was supplied by Santa Cruz Biotechnology (Santa Cruz, CA, USA).

\subsection{Determination of the optimal dosing regimen of single and combined exposures}

Chronic toxicity data evaluated in D. magna and C.dubia from exposures to single anti-neoplastic drugs (Parrella et al., 2014) were extracted to obtain a standardized format of results for all experiments. This was done by application of the isobologram method. The goal of the combined experiments is to determine qualitatively the type of interaction between drugs. To do so it is necessary to specify a reference model of interaction. As mentioned above, there are mainly two such reference models: Concentration addition (CA) and independent action (IA) also termed Bliss independence. Concentration addition is the plausible reference in case one substance can be considered a dilution of the other, while independent action applies in cases where the substances act by different pathways. Once the reference model is defined, for each effect size the curve in the concentration plane spanned by the concentrations of substance A on one axis and of substance B on the other can be computed based on the concentration-response curves from each of these substances acting alone that is expected (under the reference model) to result in the same effect. These curves are termed isobolograms (Tallarida, 2006). In order to determine the relevant isobolograms the pooled variance of responses within concentration groups was computed. Starting from concentration zero, two or three isobolograms were computed that differed each by an expected response 2 standard deviations apart. For these effect sizes the experimental concentrations were computed by dissecting the isobolograms with the diagonal of a rectangle in the concentration plane with one edge at the origin and the others at the EC50 of substance A and B, respectively. The three dissections of this line with the isobolograms determine the three optimal concentration combinations for the combined exposure experiments. Only the two lowest optimal concentrations as single exposures or in combinations were used in the experiments because the highest concentrations were too large.

\subsection{Mixture preparation}

Stock solution $(20 \mathrm{mg} / \mathrm{L})$ of each chemical was prepared in deionized water (Elix 10, Millipore, Milan, Italy) except ET that was at first dissolved in dimethyl sulphoxide (DMSO) and then diluted in deionized water $(0.02 \%, \mathrm{v} / \mathrm{v}$, in the stock solution). The test solutions were prepared by serial dilutions of the stock solutions with test media, immediately before tests. DMSO percentage in ET test solutions was lower than $0.01 \%(\mathrm{v} / \mathrm{v})$ which is a non-effective proportion as reported in international standard guideline (ISO 10706, 2000). The stability of compounds in stock solutions and test solutions was investigated (Parrella et al., 2014).

Lab work was performed in compliance with current safety guidelines and the use of personal protective equipment. Proper disposal procedures for hazardous wastes were followed.

For each test the toxicities of the binary mixtures were tested at the predicted concentrations, obtained by the isobologram method as shown in Tables 1 and 2. Based on the assumption of independent action (Bliss independence), the experiment was designed to result in equal effects of single and combined exposures at the two specified effect levels.

\subsection{Chronic toxicity testing}

Based on the chronic concentration-response data from individual drugs, a log logistic function (response at concentration $x$ : $\left.R(x)=A(x / \mu)^{ß} /\left(1+(x / \mu)^{ß}\right)\right)$ was fitted and two optimally discriminating endpoints were selected: 31.6 and 63.3 percent reduction of D. magna offspring and 44.6 and 89.2 percent reduction of $C$. dubia offspring. The concentrations expected to result in the selected responses for single and binary combinations were determined. Six binary combinations were tested: CDDP + 5-FU, IM + 5-FU, ET + 5FU, ET + CDDP, IM + ET, IM + CDDP.

The inhibition of reproduction tests on D. magna (OECD 211, 2008; ISO 10706, 2000) were performed over 21 days of exposure on young female organisms less than $24 \mathrm{~h}$ old that were from the third to fifth generation isolated from a healthy mass culture. The organisms were transferred to glass beakers (one organism in each beaker) containing $50 \mathrm{~mL}$ of sample. Crustaceans were maintained and tested in moderately fresh water (hardness $170 \mathrm{mg} / \mathrm{L}$, expressed as $\mathrm{CaCO}_{3}$; ISO 10706). All test media were exchanged every second day (semi-static conditions). The offspring produced by each parent animal were counted and removed at renewal time starting from day 9 of exposure.

The inhibition of reproduction tests on C. dubia were performed over 7 days (ISO 20665, 2008). C. dubia stock cultures were maintained in synthetic water with ISO medium (hardness $250 \mathrm{mg} / \mathrm{L}$ expressed as $\mathrm{CaCO}_{3}$ ). Females were exposed individually in beakers with 25-30 mL of test solution. All test media were exchanged five times per week (semi-static conditions). Daily, at the renewal time, the offspring produced by each parent animal were counted and removed starting from day 4 of exposure.

A combination of $5 \mathrm{~g} / \mathrm{L}$ each of yeast (Saccharomyces cerevisiae), alfalfa, and flake food (YCT), in addition to the unicellular green alga Pseudokirchneriella subcapitata $\left(10^{8}\right.$ cells/mL from cultures), provided suitable nutrition for crustaceans. The organisms were fed daily with $200 \mu \mathrm{L}$ of the YCT/algae (1/1) suspension. Ten replicates per both single and mixture concentrations were incubated at $20 \pm 1^{\circ} \mathrm{C}$ for $D$. magna and $25 \pm 1^{\circ} \mathrm{C}$ for $C$. dubia, with a $16: 8 \mathrm{~h}$ light:dark cycle (500 lux corresponding to $\sim 10 \mu \mathrm{mol}$ photons $\mathrm{s}^{-1} \mathrm{~m}^{-2}$ ). For all tests, a medium control series (negative control) was used in addition to the test series. The reproductive output of the females exposed to the cytostatics was compared to that of the negative control to determine the reduction in offspring.

\subsection{Data analysis of combined effects}

The drugs in mixtures and in single exposures were tested two times (two independent assays) for both crustaceans in ten replicates for each chronic toxicity experiment. Results were analyzed using ToxRat Professional software, Ver 2.10.05 (Alsdorf, Germany) to obtain the offspring reduction percentage. For further analysis percent reductions were arcsine transformed to remove the correlation between mean and standard deviation for data that are given as percentages and hypotheses tested by analysis of variance 
Table 1

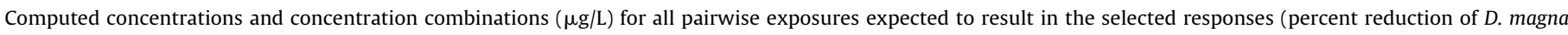
offspring).

\begin{tabular}{|c|c|c|c|c|c|}
\hline \multirow[t]{2}{*}{ Drug 1} & \multirow[t]{2}{*}{ Drug 2} & \multirow[t]{2}{*}{ \% Reduction } & \multicolumn{2}{|c|}{ Single exposure $(\mu \mathrm{g} / \mathrm{L})$} & \multirow{2}{*}{$\begin{array}{l}\text { Combined exposure }(\mu \mathrm{g} / \mathrm{L}) \\
\text { Drug } 1 / \text { Drug } 2\end{array}$} \\
\hline & & & Drug 1 & Drug 2 & \\
\hline IM & ET & 31.6 & 36.4 & 163.5 & $35.32 / 20.42$ \\
\hline IM & ET & 63.3 & 514.0 & 297.1 & 223.16/129.01 \\
\hline IM & CDDP & 31.6 & 36.4 & 0.5 & $20.83 / 0.08$ \\
\hline IM & CDDP & 63.3 & 514.0 & 2.0 & $127.90 / 0.49$ \\
\hline IM & $5-F U$ & 31.6 & 36.4 & 12.2 & $26.58 / 1.88$ \\
\hline IM & 5-FU & 63.3 & 514.0 & 36.4 & $153.67 / 10.87$ \\
\hline ET & CDDP & 31.6 & 163.5 & 0.5 & $61.43 / 0.40$ \\
\hline ET & CDDP & 63.3 & 297.1 & 2.0 & $155.00 / 1.02$ \\
\hline ET & 5-FU & 31.6 & 163.5 & 12.2 & $77.87 / 9.53$ \\
\hline ET & 5-FU & 63.3 & 297.1 & 36.4 & $165.38 / 20.23$ \\
\hline CDDP & $5-\mathrm{FU}$ & 31.6 & 0.5 & 12.2 & $0.27 / 5.00$ \\
\hline CDDP & $5-\mathrm{FU}$ & 63.3 & 2.0 & 36.4 & $0.79 / 14.66$ \\
\hline
\end{tabular}

Table 2

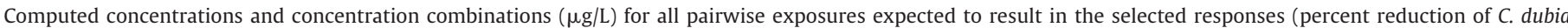
offspring).

\begin{tabular}{|c|c|c|c|c|c|}
\hline \multirow[t]{2}{*}{ Drug 1} & \multirow[t]{2}{*}{ Drug 2} & \multirow[t]{2}{*}{ \% Reduction } & \multicolumn{2}{|c|}{ Single exposure $(\mu \mathrm{g} / \mathrm{L})$} & \multirow{2}{*}{$\begin{array}{l}\text { Combined exposure }(\mu \mathrm{g} / \mathrm{L}) \\
\text { Drug } 1 / \text { Drug } 2\end{array}$} \\
\hline & & & Drug 1 & Drug 2 & \\
\hline IM & ET & 44.6 & 36.6 & 196.5 & $18.3 / 97.3$ \\
\hline $\mathrm{IM}^{\mathrm{a}}$ & ET & 89.2 & & & \\
\hline IM & CDDP & 44.6 & 36.6 & 10.7 & $7.0 / 2.1$ \\
\hline $\mathrm{IM}^{\mathrm{a}}$ & CDDP & 89.2 & & & \\
\hline IM & 5-FU & 44.6 & 36.6 & 2.5 & $8.1 / 0.6$ \\
\hline $\mathrm{IM}^{\mathrm{a}}$ & 5-FU & 89.2 & & & \\
\hline ET & CDDP & 44.6 & 160.7 & 12.3 & $41.97 / 11.10$ \\
\hline ET & CDDP & 89.2 & 473.7 & 125.3 & $189.80 / 50.20$ \\
\hline ET & $5-\mathrm{FU}$ & 44.6 & 160.7 & 3.8 & $76.10 / 2.92$ \\
\hline ET & 5-FU & 89.2 & 473.7 & 18.2 & $218.05 / 8.37$ \\
\hline CDDP & 5-FU & 44.6 & 12.3 & 3.8 & $8.55 / 1.24$ \\
\hline CDDP & 5-FU & 89.2 & 125.3 & 18.2 & $40.19 / 5.83$ \\
\hline
\end{tabular}

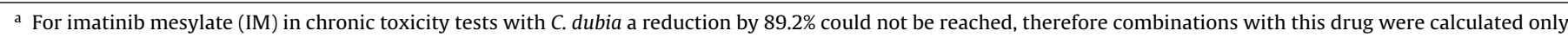
for the lower responses.

(ANOVA). Total deviation from expected equality of reductions under the assumption of IA (Bliss independence) was tested by the overall ANOVA result for the factor exposure type, while each single component was tested against the binary mixture by linear contrasts. Because concentrations of each substance tested under single exposure conditions and for combined experiments were chosen to result in the same expected effect, testing the hypothesis of equality between expected means is at the same time testing the hypothesis of independent action. On the other hand, a significant deviation from equality of effect sizes points towards antagonism or synergism depending on whether the combined effect is smaller or larger than the one of the single compound, respectively. Homogeneity of variances was tested by Bartlett tests, normality of residuals were tested by Kolmogorov-Smirnov test with Lilliefors corrected $p$-values. These analyses were performed by Statistica 10.0 (StatSoft Inc., 2011). For hypothesis testing $p$-values below 0.05 were considered significant.

\section{Results and discussion}

The consumption patterns and the physico-chemical properties of the anti-neoplastic drugs have been recently provided (Kosjek and Heath, 2011; Besse et al., 2012; Xie, 2012; Zhang et al., 2013) as well as some information about their toxic effects on non-target organisms (Brezovšek et al., 2014; Parrella et al., 2014; Pichler et al., 2014). Among the anti-neoplastic drugs studied, IM is a more recently introduced drug in chemotherapy and information concerning its environmental occurrence and fate is lacking (Negreira et al., 2013). While 5-FU, CDDP and ET are used in combination in cancer treatments and they are expected to be in mixture in waste waters, IM is generally administered alone but for its high consumption (Besse et al., 2012; Kümmer et al., 2009) it cannot be excluded in mixture with other anticancer drugs. Concentrations of such compounds in surface waters are difficult to predict depending on their consumption levels, excretion rates, removal rates, and local factors (Toolaram et al., 2014). The lack of knowledge about environmental fate for compounds interacting with DNA is a significant limitation because for these substances no safe level can be defined, especially when they are present in combinations. Furthermore, genotoxic alterations could be of high ecotoxicological relevance affecting the reproductive capability at the whole organism level (Jha, 2008). In order to evaluate the possible interactions of the selected anti-neoplastic drugs in mixtures, the toxicities of binary combinations on two crustaceans were statistically compared to those of single compounds; $D$. magna and C. dubia were selected for their worldwide distribution in freshwater and their use in standard toxicity procedures.

In $D$. magna, the concentrations computed by the isobologram method were predicted to cause a 31.6 and $63.3 \%$ reduction in the number of offspring after 21 days of exposure. Single compound and mixture results are described in Fig. 1. Inhibition percentages of single compounds were close to expected values for the lower concentrations. For the higher concentrations there were higher responses than expected for 5-FU and ET as shown in Fig. 1a. All results of binary combinations (Fig. 1b) for the lower concentrations were not different from those of single compounds $(p>0.05)$, indicating Bliss independent action (Table 3 ). For the higher concentrations, significant deviations from independence occurred in two mixtures with IM, IM + ET vs. ET $(p<0.001)$ and IM + CDDP $v s . \operatorname{CDDP}(p<0.05)$. Both interactions were antagonistic suggesting 

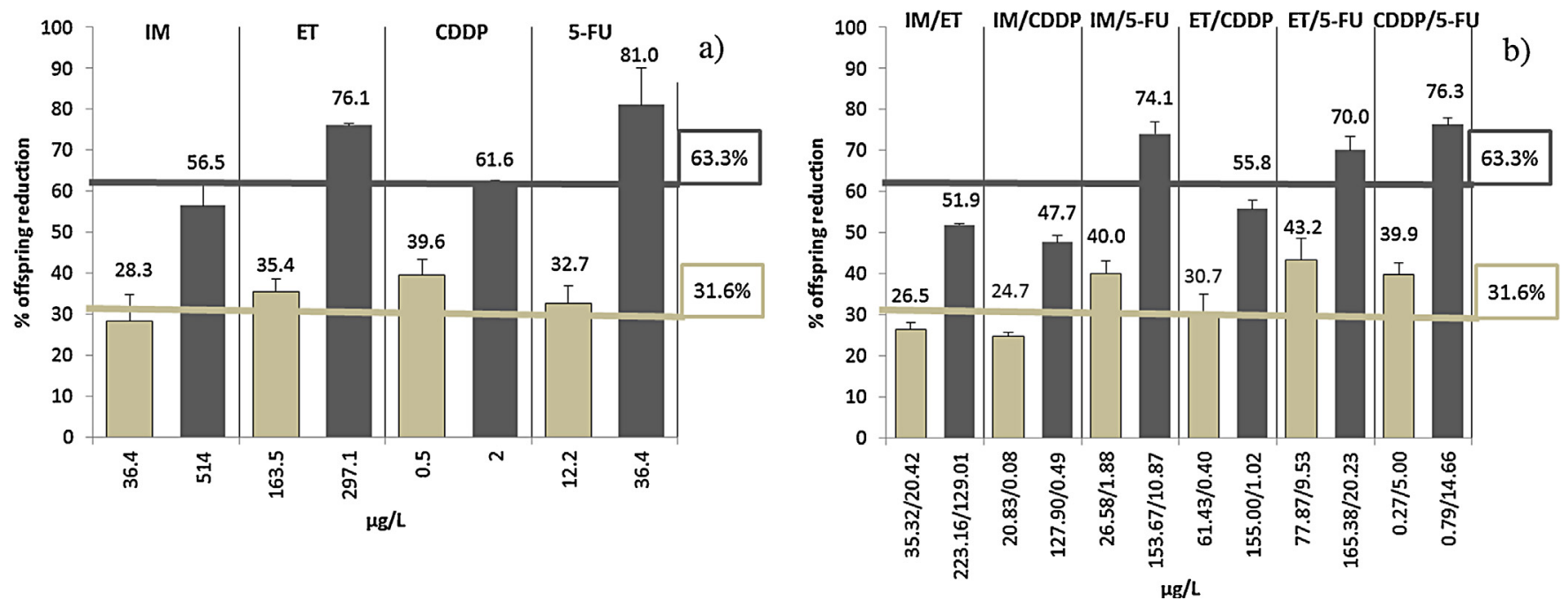

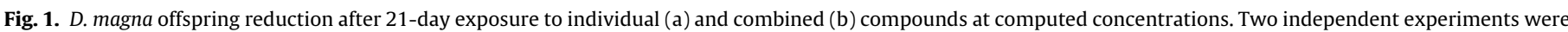

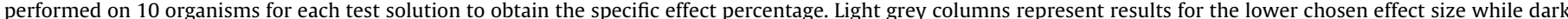

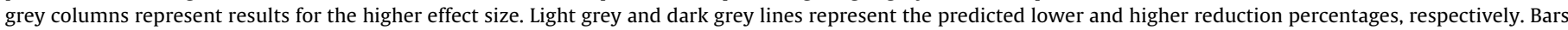
are means \pm SE. Concentrations in $\mu \mathrm{g} / \mathrm{L}$ are shown on the $x$ axis, means of offspring reductions are indicated above the columns.

\section{Table 3}

Results of ANOVA and overview of results of combined experiments in relation to the Bliss independence reference model for $D$. magna chronic toxicity test. Comparison between each single compound and respective binary mixture at the two predicted effects causing 31.6 and $63.3 \%$ offspring reduction after 21 days exposure. A significant difference indicates deviation from Bliss independence.

\begin{tabular}{|c|c|c|c|}
\hline Effect \% & Comparison & $p$-Value & Interaction \\
\hline \multirow{2}{*}{31.6} & CDDP vs CDDP/5-FU & 0.794 & \multirow{2}{*}{ Bliss independence } \\
\hline & 5-FU vs CDDP/5-FU & 0.386 & \\
\hline \multirow{2}{*}{63.3} & CDDP vs CDDP/5-FU & 0.006 & \multirow{2}{*}{ Bliss independence ${ }^{a}$} \\
\hline & 5-FU vs CDDP/5-FU & 0.124 & \\
\hline \multirow{2}{*}{31.6} & CDDP vs ET/CDDP & 0.740 & \multirow{2}{*}{ Bliss independence } \\
\hline & ET vs ET/CDDP & 0.859 & \\
\hline \multirow{2}{*}{63.3} & CDDP vs ET/CDDP & 0.901 & \multirow{2}{*}{ Bliss independence } \\
\hline & ET vs ET/CDDP & 0.071 & \\
\hline \multirow{2}{*}{31.6} & CDDP vs IM/CDDP & 0.354 & \multirow{2}{*}{ Bliss independence } \\
\hline & IM vs IM/CDDP & 0.942 & \\
\hline \multirow{2}{*}{63.3} & CDDP vs IM/CDDP & 0.020 & \multirow{2}{*}{ Antagonism } \\
\hline & IM vs IM/CDDP & 0.156 & \\
\hline \multirow{2}{*}{31.6} & 5-FU vs ET/5-FU & 0.289 & \multirow{2}{*}{ Bliss independence } \\
\hline & ET vs ET/5-FU & 0.431 & \\
\hline \multirow{2}{*}{63.3} & 5-FU vs ET/5-FU & 0.232 & \multirow{2}{*}{ Bliss independence } \\
\hline & ET vs ET/5-FU & 0.754 & \\
\hline \multirow[b]{2}{*}{31.6} & 5-FU vs IM/5-FU & 0.333 & \multirow{2}{*}{ Bliss independence } \\
\hline & IM $v s$ IM $/ 5-F U$ & 0.116 & \\
\hline \multirow{2}{*}{63.3} & 5-FU vs IM/5-FU & 0.481 & \multirow{2}{*}{ Bliss independence $^{a}$} \\
\hline & IM vs IM/5-FU & $<0.001$ & \\
\hline \multirow{2}{*}{31.6} & ET vs IM/ET & 0.801 & \multirow{2}{*}{ Bliss independence } \\
\hline & IM vs IM/ET & 0.655 & \\
\hline \multirow{2}{*}{63.3} & ET $v s$ IM/ET & $<0.001$ & \multirow{2}{*}{ Antagonism } \\
\hline & $\mathrm{IM} v s \mathrm{IM} / \mathrm{ET}$ & 0.601 & \\
\hline
\end{tabular}

a Despite the significant difference between single and combined exposure, due to the higher than expected single exposure effect for 5-FU, the result does not indicate a deviation from independency.

that IM action interfered with the activity of ET and CDDP. Imatinib mesylate is an anticancer drug inhibiting numerous tyrosine kinases such as Abl and Bcr-Abl tyrosine kinase, the tyrosine kinase receptor for stem cell factor, c-kit and PDGF-r (Kübler et al., 2005). Etoposide, as topoisomerase II inhibitor, interferes with DNA replication forming complexes that avoid DNA relegation (Xie, 2012) while Cisplatin is an alkylating agent that activates apoptosis pathway in the target tumour cells causing DNA inter-strand cross-links and adducts (Xie, 2012). As these compounds act on different molecular targets, it is difficult to understand the mechanism of the observed antagonistic interactions. Several studies on the combined effects of these drugs describe controversial results going from antagonistic to synergistic effects when performed on different cancer cell lines with the aim of modifying the chemotherapeutic strategies (Wozniak et al., 2004; Kübler et al., 2005).

All other binary combinations did not show significant deviations from Bliss independence. ET + CDDP showed a tendency for antagonistic effects $(p=0.071)$ after recalculation of the expected effect that became necessary due to the differences in the predicted to the observed concentration-response of the single compounds (Table 3). Combination effects including the higher concentration of 5 -FU were higher than predicted but this is to be expected because of the higher reduction percentage of exposure to single 5-FU. In the combination experiments, the concentration-response was slightly steeper than in the previous experiments resulting in single and combined effects of 5-FU above the expected level (data not shown).

In $C$. dubia, the concentrations computed by the isobologram method were predicted to cause 44.6 and $89.2 \%$ reduction in the number of offspring after 7 days of exposure. The results of single compounds and mixtures are shown in Fig. 2. Generally, values for single compounds were very close to the predicted reductions (Fig. 2a). In none of the experiments a deviation from the expected effects was observed. IM, differently from the other drugs investigated, did not show a rapid increase in concentration/effect relationship in single exposure (Parrella et al., 2014) and was tested only at the lower concentration (expected reduction $44.6 \%$ ) because the reduction by $89.2 \%$ could not be reached. In C. dubia, at the lower effect level, all binary mixture results (Fig. 2b) were not significantly different $(p>0.05)$ from those of single compounds, indicating Bliss independent action (Table 4) except IM + CDDP which compared to exposure to single IM showed a tendency $(p=0.076)$ to synergism. At the higher effect level (expected reduction 89.2\%) ET + CDDP and ET +5 -FU showed lower effects than single compounds, indicating antagonistic interaction ( $p<0.001$, Table 4$)$.

The similar toxicological sensitivities of $D$. magna and $C$. dubia, already demonstrated in previous studies (Constantine and Huggett, 2010; Parrella et al., 2014) were confirmed also in the present study, in fact most of mixtures showed a Bliss independence interaction in both organisms. Each cytostatic, when in mixture, likely exerted effects on non-target organisms at concentrations not depending on the other cytostatic (Katsnelson et al., 2011). This interaction, also known as heteroadditivity, was 

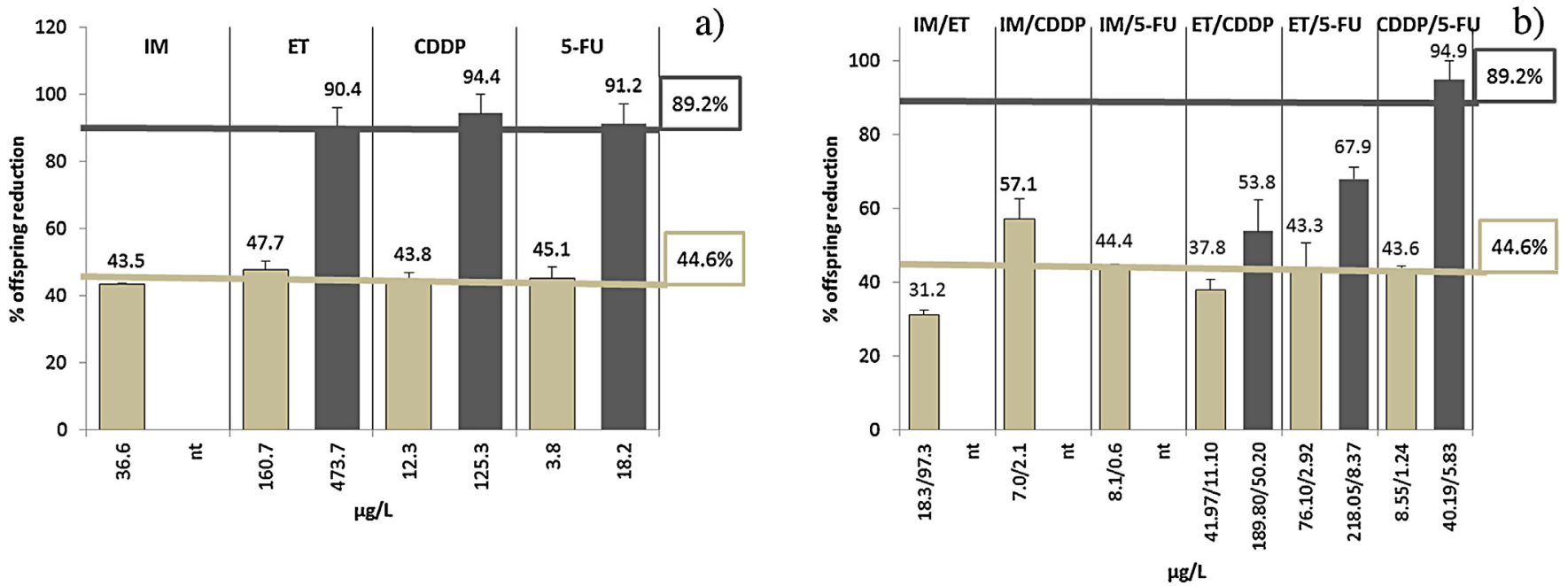

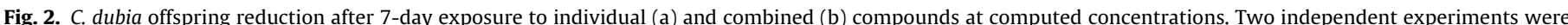

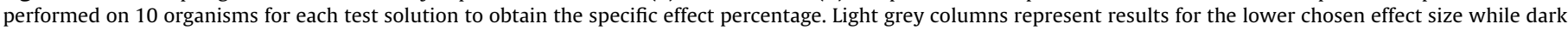

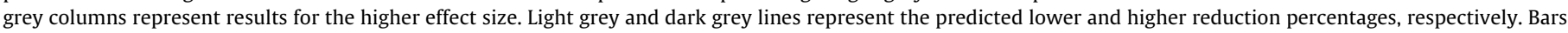
are means \pm SE. Concentrations in $\mu \mathrm{g} / \mathrm{L}$ are shown on the $x$ axis, means of offspring reductions are indicated above the columns.

Table 4

Results of ANOVA and overview of results of combined experiments in relation to the Bliss independence reference model for $C$. dubia chronic toxicity test. Comparison between each single compound and respective binary mixture at the two predicted effects causing 44.6 and $89.2 \%$ offspring reduction after 7 days exposure. IM and the respective mixtures were tested only at the lower effect\% because the reduction by $89.2 \%$ could not be reached. A significant difference indicates deviation from Bliss independence.

\begin{tabular}{|c|c|c|c|}
\hline Effect \% & Comparison & $p$-Value & Interaction \\
\hline \multirow{2}{*}{44.6} & CDDP vs CDDP/5-FU & 0.739 & \multirow{2}{*}{ Bliss independence } \\
\hline & 5-FU vs CDDP/5-FU & 0.354 & \\
\hline \multirow{2}{*}{89.2} & CDDP vs CDDP/5-FU & 0.910 & \multirow{2}{*}{ Bliss independence } \\
\hline & 5-FU vs CDDP/5-FU & 0.484 & \\
\hline \multirow{2}{*}{44.6} & CDDP vs ET/CDDP & 0.654 & \multirow{2}{*}{ Bliss independence } \\
\hline & $\mathrm{ET} v s \mathrm{ET} / \mathrm{CDDP}$ & 0.851 & \\
\hline \multirow{2}{*}{89.2} & CDDP vs ET/CDDP & $<0.001$ & \multirow{2}{*}{ Antagonism } \\
\hline & $\mathrm{ET} v s \mathrm{ET} / \mathrm{CDDP}$ & $<0.001$ & \\
\hline \multirow{2}{*}{44.6} & CDDP vs IM/CDDP & 0.237 & \multirow{2}{*}{ Synergism (tendency) } \\
\hline & IM vs IM/CDDP & 0.076 & \\
\hline \multirow{2}{*}{44.6} & 5-FU vs ET/5-FU & 0.436 & \multirow{2}{*}{ Bliss independence } \\
\hline & ET vs 5- ET/5-FU & 0.654 & \\
\hline \multirow{2}{*}{89.2} & 5-FU vs ET/5-FU & $<0.001$ & \multirow{2}{*}{ Antagonism } \\
\hline & ET vs ET/5-FU & $<0.001$ & \\
\hline \multirow{2}{*}{44.6} & 5-FU vs IM/5-FU & 0.572 & \multirow{2}{*}{ Bliss independence } \\
\hline & IM vs IM/5-FU & 0.805 & \\
\hline \multirow{2}{*}{44.6} & ET vs IM/ET & 0.131 & \multirow{2}{*}{ Bliss independence } \\
\hline & IM vs IM/ET & 0.190 & \\
\hline
\end{tabular}

not demonstrated in D. magna by IM + ET and IM + CDDP showing antagonism at higher concentration even if it was not possible to verify the same effect in $C$. dubia because the higher effect level could not be tested. Antagonism was observed also in C. dubia for ET + CDDP and ET + 5-FU. The ET + CDDP antagonistic effect was found also by Brezovšek and co-authors (2014) on the unicellular alga Pseudokirchneriella subcapitata and the cyanobacterium Synechoccus leopoliensis. As reported in this study, the antagonistic effect observed might be caused by the suppression of the toxic effect of CDDP due to ET. So far, an antagonistic mode of action of topoisomerase inhibitor (topotecan) in mixture with CDDP was found for prokaryotes where topotecan was able to block the DNA replication forks reducing the CDDP DNA interaction. The antagonistic activity of ET in binary mixture could be exerted also in presence of 5-FU. The latter is a pyrimidine analogue interfering with nucleic acid metabolism. 5-FU is converted to different metabolites, especially fluorodeoxyuridine monophosphate, which forms a stable complex with thymidylate synthase. Thus, this drug interferes with DNA phase S synthesis (Xie, 2012). Probably, the antagonistic effect found for the mixture ET +5-FU could be due to the block of the incorporation of 5-FU metabolites into DNA (Jia et al., 2009).

Traditional risk assessment pays particular attention to consumption patterns and resulting environmental concentrations of drugs but it is not fully focused on bioavailability and bioaccessibility of environmental residues (Caldwell et al., 2014). More information about physico-chemical properties and environmental fate of such compounds would be necessary. 5-FU, CDDP and ET are highly mobile in the aquatic environment $\left(\log K_{\text {ow }}<1\right)$. Furthermore, the fraction of unchanged 5-FU excretion is very low because it is a pro-drug with metabolites, which are more active than the parent compound, even if 5-FU is found in sewers because it is the most administered anticancer drug in the world (Kosjek et al., 2013). 5-FU, CDDP and ET are often administered in combination for cancer treatments and 5-FU and CDDP were found to be chronically toxic towards non-target organisms of the aquatic chain (Brezovšek et al., 2014; Parrella et al., 2014). Then, a risk at long-term exposure for 5-FU and CDDP could not be excluded also for their presence in the hospital effluents at concentrations in the range $<5 \mathrm{ng} / \mathrm{L}-$ $124 \mu \mathrm{g} / \mathrm{L}$ for 5-FU and 2-250 $\mu \mathrm{g} / \mathrm{L}$ for Pt compounds (Mahnik et al., 2004, 2007; Lenz et al., 2007; Kovalova et al., 2009; Kosjek et al., 2013). IM and ET seem to be of less environmental concern when in mixture considering the effects obtained at high concentrations and the antagonism implicated. Nevertheless a risk cannot be excluded as IM has low mobility and can absorb onto sludges and sediments with possibility of biomagnification, as its $\log K_{\text {ow }}=3$ (Xie, 2012); furthermore, IM is among the most administered anti-neoplastic drugs, its consumption is constantly increasing and no information exists about its fate in the environment (Toolaram et al., 2014).

\section{Conclusion}

In light of these results, mixtures generally showed Bliss independence producing additive effects of environmental concern, since such compounds are present as a cocktail in real samples. Furthermore, the same effect levels produced by single exposures, are obtained in mixtures at much lower concentrations. In this study, the statistical models CA and IA confirmed their good predictive power even if, as shown, they could overestimate or underestimate the toxicity of mixtures. Then, these models should be more often 
combined to experimental data and physico-chemical properties to have an overall knowledge about the real environmental risk of such compounds.

\section{Acknowledgement}

This work was financially supported by the EU through the EU FP7 project CytoThreat (Fate and effects of cytostatic pharmaceuticals in the environment and the identification of biomarkers for an improved risk assessment on environmental exposure. Grant agreement no. 265264).

\section{References}

Besse, J.P., Latour, J.F., Garric, J., 2012. Anticancer drugs in surface waters: what can we say about the occurrence and environmental significance of cytotoxic, cytostatic and endocrine therapy drugs? Environ. Int. 39, 73-86.

Brezovšek, P., Eleršek, T., Filipič, M., 2014. Toxicities of four anti-neoplastic drugs and their binary mixtures tested on the green alga Pseudokirchneriella subcapitata and the cyanobacterium Synechococcus leopoliensis. Water Res. 52, 168-177.

Caldwell, D.J., Mastrocco, F., Margiotta-Casaluci, L., Brooks, B.W., 2014. An integrated approach for prioritizing pharmaceuticals found in the environment for risk assessment, monitoring and advanced research. Chemosphere, http://dx.doi.org/10.1016/j.chemosphere.2014.01.021.

Constantine, L.A., Huggett, D.B., 2010. A comparison of the chronic effects of human pharmaceuticals on two cladocerans, Daphnia magna and Ceriodaphnia dubia. Chemosphere 80, 1069-1074, http://dx.doi.org/10.1016/ j.chemosphere.2014.01.013.

Fent, K., Escher, C., Caminada, D., 2006. Estrogenic activity of pharmaceuticals and pharmaceutical mixtures in a yeast reporter gene system. Reprod. Toxicol. 22, 175-185.

Galus, M., Jeyaranjaan, J., Smith, E., Li, H., Metcalfe, C., Wilson, J.Y., 2013. Chronic effects of exposure to a pharmaceutical mixture and municipal wastewater in zebrafish. Aquat. Toxicol. 132-133, 212-222.

Greco, W., Unkelbach, H.D., Pöch, G., Sühnel, J., Kundi, M., Bödeker, W., 1992. Consensus on concepts and terminology for combined-action assessment: the Saariselkä agreement. Arch. Complex Environ. Stud. 4 (3), 65-69.

ISO 10706, 2000. Water Quality-Determination of Long Term Toxicity of Substances to Daphnia magna Straus (Cladocera, Crustacean). International Organization for Standardization, Geneva, Switzerland.

ISO 20665, 2008. Water Quality-Determination of Chronic Toxicity to Ceriodaphnia dubia in 7 Days-Population Growth Inhibition Test. International Organization for Standardization, Geneva, Switzerland.

Jha, A.N., 2008. Ecotoxicological applications and significance of the comet assay. Mutagenesis 23 (3), 207-221.

Jia, J., Zhu, F., Ma, X., Cao, Z.W., Li, Y.X., Chen, Y.Z., 2009. Mechanisms of drug combinations: interaction and network perspectives. Nat. Rev. Drug Discov. 8, 111-128.

Katsnelson, B.A., Privalova, L.I., Varaksin, A.N., Kazmer, J.I., Kireyeva, E.P., Panov, V.G., 2011. An approach to characterizing the type of combined environmental toxicity based on epidemiologically assessed exposure-response relationships. Open Epidemiol. J. 4, 60-69.

Kosjek, T., Heath, E., 2011. Occurrence, fate and determination of cytostatic pharmaceuticals in the environment. Trends Anal. Chem. 30, 1065-1087.
Kosjek, T., Perko, S., Žigon, D., Heath, E., 2013. Fluorouracil in the environment: analysis, occurrence, degradation and transformation. J. Chromatogr. A. 1290, 62-72.

Kovalova, L., McArdell, C.S., Hollender, J., 2009. Challenge of high polarity and low concentrations in analysis of cytostatics and metabolites in wastewater by hydrophilic interaction chromatography/tandem mass spectrometry. J. Chromatogr. A 1216, 1100-1108.

Kübler, H.R., van Randenborgh, H., Treiber, U., Wutzler, S., Battistel, C., Lehmer, A. Wagenpfeil, S., Hartung, R., Paul, R., 2005. In vitro cytotoxic effects of imatinib in combination with anticancer drugs in human prostate cancer cell lines. Prostate 63 (4), 385-394.

Kümmer, K., Schuster, A., Haiss, A., Günther, A., Jacobs, J., Mohring, S., Hamsher, G. Simon, D., 2009. Umweltrisikobewertung von Zytostatika. Umweltbundesamt, f. UBA-FB001256, Dessau-Roßlau.

Kümmerer, K., 2009. The presence of pharmaceuticals in the environment due to human use - present knowledge and future challenges. J. Environ. Manage. 90, 2354-2366.

Lenz, K., Mahnik, S.N., Weissenbacher, N., Mader, R.M., Krenn, P., Hann, S., Koellensperger, G., Uhl, M., Knasmueller, S., Ferk, F., Bursh, W., Fuerhacker, M., 2007. Monitoring, removal and risk assessment of cytostatic drugs in hospital wastewater. Water Sci. Technol. 56 (12), 141-149.

Mahnik, S.N., Rizovski, B., Fürhacker, M., Mader, R.M., 2004. Determination of 5 fluorouracil in hospital effluents. Anal. Bioanal. Chem. 380, 31-35.

Mahnik, S.N., Lenz, K., Weissenbacher, N., Mader, R.M., Fuerhacker, M., 2007. Fate of 5-fluorouracil, doxorubicin, epirubicin, and daunorubicin in hospital wastewater and their elimination by activated sludge and treatment in a membrane-bio-reactor system. Chemosphere 65 (1), 30-37.

Negreira, N., López de Alda, M., Barceló, D., 2013. On-line solid phase extractionliquid chromatography-tandem mass spectrometry for the determination of 17 cytostatics and metabolites in waste, surface and ground water samples. J. Chromatogr. A 1280, 64-74.

OECD 211, 2008. Daphnia Magna Reproduction Test. Organization for Economic Cooperation and Development, Paris.

Parrella, A., Lavorgna, M., Criscuolo, E., Russo, C., Fiumano, V., Isidori, M., 2014 Acute and chronic toxicity of six anticancer drugs on rotifers and crustaceans. Chemosphere 115, 59-66.

Pichler, C., Filipič, M., Kundi, M., Rainer, B., Knasmueller, S., Mišík, M. 2014. Assessment of genotoxicity and acute toxic effect of the imatinib mesylate in plant bioassays. Chemosphere, http://dx.doi.org/10.1016/j. chemosphere.2014.01.010.

Shi, S., Yao, W., Xu, J., Long, J., Liu, C., Yu, X., 2012. Combinational therapy: new hope for pancreatic cancer? Cancer Lett. 317 (2), 127-135.

Statsoft Inc., 2011. STATISTICA (data analysis software system), ver. 10.0. Statsoft Inc.ed., Tulsa, OK, USA.

Tallarida, R.J., 2006. An overview of drug combination analysis with isobolograms J. Pharmacol. Exp. Ther. 319 (1), 1-7.

Toolaram, A.P., Kümmerer, K., Schneider, M., 2014. Environmental risk assessment of anti-cancer drugs and their transformation products: a focus on their genotoxicity characterization-state of knowledge and short comings. Mutat. Res. http://dx.doi.org/10.1016/j.mrrev.2014.02.001.

Wozniak, K., Czechowska, A., Blasiak, J., 2004. Cisplatin-evoked DNA fragmentation in normal and cancer cells and its modulation by free radical scavengers and the tyrosine kinase inhibitor STI571. Chem. Biol. Interact. 147 (3), 309-318.

Xie, H., 2012. Occurrence, ecotoxicology, and treatment of anticancer agents as water contaminants. J. Environ. Anal. Toxicol. S2, 1-11.

Zhang, J., Chang, V.W.C., Giannis, A., Wang, J.Y., 2013. Removal of cytostatic drugs from aquatic environment: a review. Sci. Total Environ. 445-446, 281-298. 